\title{
Effect of luteolin on the methylation status of the OPCML gene and cell growth in breast cancer cells
}

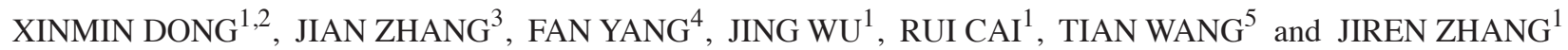 \\ ${ }^{1}$ Department of Oncology, Zhujiang Hospital, Southern Medical University, Guangzhou, Guangdong 510280; \\ ${ }^{2}$ Department of Oncology, The Affiliated People's Hospital of Inner Mongolia Medical University, Hohhot, Inner \\ Mongolia 010010; ${ }^{3}$ Department of Radiotherapy, The Affiliated People's Hospital of Inner Mongolia Medical University, \\ Hohhot, Inner Mongolia 010017; ${ }^{4}$ Department of Basic Medicine, Xiangnan University, Chenzhou, Hunan 423000; \\ ${ }^{5}$ Department of Hematology and Oncology, Yan'an People's Hospital, Yan'an, Shaanxi 716000, P.R. China
}

Received December 6, 2017; Accepted May 24, 2018

DOI: $10.3892 /$ etm.2018.6526

\begin{abstract}
The present study aimed to investigate the effect of luteolin on the methylation of opioid binding protein/cell adhesion molecule (OPCML) in breast cancer cells, as well as its underlying mechanism of action. Human breast cancer cell lines BT474 and MCF-7 were cultured in RPMI-1640 medium supplemented with $10 \%$ fetal bovine serum. The cells were treated with $0-30 \mu \mathrm{mol} / 1$ luteolin prior to investigation. Reverse transcription-quantitative polymerase chain reaction and western blot analysis were used to determine the mRNA and protein expression, respectively. High performance liquid chromatography and electrosprary ionization-mass spectrometry was used to analyze the methylation of the OPCML promoter region and whole genome. The methylation activity in the cell nucleus was determined using a DNA methyltransferase catalytic test. ELISA analysis was used to detect changes in the activity of transcription factors $\mathrm{Sp} 1$ and nuclear factor (NF)- $\mathrm{kB}$. An MTT assay was performed to determine cell proliferation, while flow cytometry was used to detect cell cycle stage and apoptosis. Luteolin effectively upregulated the expression of OPCML in breast cancer cells. Luteolin activated OPCML by reducing intracellular methylation levels. Luteolin downregulated intracellular methylation levels by decreasing Sp1 and NF- $\mathrm{kB}$ activities. Luteolin affected the expression of DNMT1 and OPCML by downregulating Sp1 activity. Luteolin inhibited the proliferation and induced the apoptosis of BT474 and MCF-7 cells. The results of the present study suggest that luteolin inhibits the growth of breast cancer cells by decreasing the methylation and upregulating the expression of the OPCML gene.
\end{abstract}

Correspondence to: Dr Jiren Zhang, Department of Oncology, Zhujiang Hospital, Southern Medical University, 253 Industrial Road, Guangzhou, Guangdong 510280, P.R. China

E-mail: zhangjiren2015@163.com

Key words: breast cancer, luteolin, opioid binding protein/cell adhesion molecule

\section{Introduction}

Breast cancer is one of the most serious threats to women's health (1). In Western countries, breast cancer cases account for about $1 / 3$ of all new cancer cases in women, ranking the first in women with malignant tumors (2). The incidence of breast cancer is increasing at an annual rate of $2 \%$ in coastal cities of China (3). The emergence of multidisciplinary treatment methods such as targeted therapy and the overall survival of patients with breast cancer has been prolonged, but the survival rate of patients with advanced breast cancer is still not significantly improved (4). Understanding the mechanism of breast cancer is helpful for further improving the level of clinical treatments of breast cancer.

The occurrence of tumors is caused by the activation of oncogenes and inactivation of tumor-suppressor genes. The changes of genes include two aspects, genetics and epigenetics. Epigenetics can affect gene transcription and translation, but DNA sequences are not changed. Epigenetics can affect signal transduction pathway of single cells for several times at different sites, leading to disorders of cell structure and internal environment (5). Epigenetic alterations include DNA methylation, histone modification, chromatin remodeling and RNA interference $(6,7)$. Hypermethylation of promoter region of tumor suppressor genes is the most important research area, because it occurs at the early stage of malignant tumor formation. DNA methylation is the best understood and important epigenetic modification, and it converts cytosine to 5-methylcytocine under the action of DNA methyl transferases (DNMTs). Aberrant methylation of DNA can lead to the activation of oncogenes and inactivation of tumor-suppressor genes, being closely related to the occurrence of multiple tumors $(7,8)$.

Opioid binding protein/cell adhesion molecule (OPCML) is a novel tumor-suppressor gene located in the 11q chromosome segment. The main function of OPCML is to regulate cell adhesion and recognition, and it plays a role in inhibiting tumor growth through the activation of relevant ion channels and adenylate cyclase (9). The expression and deficiency of OPCML gene are closely related to the occurrence and development of many tumors $(10,11)$. The silencing of OPCML 
gene in human breast cancer cells is related to its methylation status (12).

Luteolin is a common natural flavonoid compound, that is rich in celery, green pepper and dandelion leaves. It has a variety of biological activities, such as anti-oxidation, anti-inflammation, anti-depression, anti-convulsion, anti-anxiety, anti-allergy, and immunity improvement (13). Studies show that luteolin can inhibit the growth of tumor cells through a variety of mechanisms in gastric cancer and ovarian cancer $(14,15)$, and effectively improves the methylation status of various tumor cells (16). These suggest that luteolin is a potential antitumor adjuvant drug. In the present study, we evaluate whether luteolin is able to restore OPCML activity in breast cancer cells, and investigate the molecular mechanism of its low methylation activity.

\section{Materials and methods}

Cells. Human breast cancer BT474, MCF-7 and MDA-MB-231 cells were purchased from American Type Culture Collection (Manassas, VA, USA), and cultured in RPMI-1640 medium (Mediatech, Manassas, VA, USA) supplemented with $10 \%$ fetal bovine serum in a humidified incubator with $5 \% \mathrm{CO}_{2}$. The number of passages of all cells was strictly controlled and mycoplasma contamination was regularly monitored.

MTT assay. MTT assay was used to determine the effect of luteolin on cell activity. Briefly, $100 \mu 1$ medium containing $3 \times 10^{3}$ cells were inoculated in a 96-well plate in triplicate wells overnight. Then, the medium was replaced with RPMI-1640 supplemented with $5 \%$ fetal bovine serum, and the cells were incubated with different concentrations of luteolin for $72 \mathrm{~h}$.

Western blotting of protein expression and phosphorylation. Cells were washed with phosphate-buffered saline and used for protein extraction from nucleus and cytoplasm. After determination of protein concentrations, protein samples $(50 \mu \mathrm{g})$ were mixed with equal volume of $2 \mathrm{X}$ sodium dodecyl sulfate loading buffer before denaturation in boiling water bath for $5 \mathrm{~min}$. Afterwards, $20 \mu \mathrm{l}$ samples were subjected to sodium dodecyl sulfate-polyacrylamide gel electrophoresis at $100 \mathrm{~V}$. The resolved proteins were transferred to polyvinylidene difluoride membranes on ice $(300 \mathrm{~mA}, 1.5 \mathrm{~h})$ and blocked with $5 \%$ skimmed milk at room temperature for $2 \mathrm{~h}$. Then, the membranes were incubated with rabbit anti-human polyclonal OPCML primary antibody (ab100923; Abcam, Cambridge, UK), anti-phospho-Histone H2A.X (Ser139) antibody (2577), total H2A.X antibody (2595), cleaved caspase-8 antibody (9496) and cleaved PARP antibody (9541; all from Cell Signaling Technology, Inc., Beverly, MA, USA) and anti-GAPDH (G9545; Sigma-Aldrich, St. Louis, MO, USA) at $4^{\circ} \mathrm{C}$ overnight. After extensive washing with phosphate-buffered saline with Tween-20 for 3 times of $15 \mathrm{~min}$, the membranes were incubated with horseradish peroxidase-conjugated secondary antibody for $1 \mathrm{~h}$ at room temperature before washing with phosphate-buffered saline with Tween-20 for 3 times of $15 \mathrm{~min}$. Then, the membrane was developed with enhanced chemiluminescence detection kit (EMD Millipore, Billerica, MA, USA) for imaging (VL Chemi-Smart 3000; Viogene-Biotek Corp., Sunnyvale, CA,
USA). The relative content of target protein was expressed against GAPDH.

Reverse transcription-quantitative polymerase chain reaction (RT-qPCR). Total RNA was extracted using TRIzol reagent (Thermo Fisher Scientific, Inc., Waltham, MA, USA). cDNA was obtained by reverse transcription using Promega's reverse transcription system (Promega Corporation, Madison, WI, USA). Then, $10 \mathrm{mmol} / 1$ forward and reverse primers and SYBR were added for RT-qPCR amplification on ABI 7500 (both from Thermo Fisher Scientific, Inc.). The primers for OPCML were 5'-GGGTCTGTGGGTACCTGTTC-3' (forward) and 5'-TAT GGACCACTTGTCATTCC-3' (reverse); and for $\beta$-actin were 5'-GTCTTCCCCTCCATCGTG-3' (forward) and 5'-AGGGTG AGGATGCCTCTCTT-3' (reverse). qPCR protocol was: Initial denaturation at $95^{\circ} \mathrm{C}$ for $2 \mathrm{~min} ; 32$ cycles of $94^{\circ} \mathrm{C}$ for $30 \mathrm{sec}$, $55^{\circ} \mathrm{C}$ for $30 \mathrm{sec}$, and $72^{\circ} \mathrm{C}$ for $30 \mathrm{sec}$; final elongation at $72^{\circ} \mathrm{C}$ for $5 \mathrm{~min}$. The $2^{-\Delta \Delta \mathrm{Cq}}$ method was used to calculate the relative expression of target mRNA against $\beta$-actin. All samples were measured in triplicate, and mean values were considered for comparative analysis.

Determination of methylation activity. Cells were washed with $1 \mathrm{ml}$ precooled phosphate-buffered saline and collected. Then, the cells were centrifuged at $800 \mathrm{rpm}$ and $4^{\circ} \mathrm{C}$ for $5 \mathrm{~min}$ before removing supernatants. Total nuclear proteins were extracted from BT474 or MCF-7 cells using protein extraction kit (Pierce, Rockford, IL, USA). According to the manufacturer's manual, DNMT analysis kit (Epigentek Group Inc., Brooklyn, NY, USA) was used to determine methylation activity. Micropores in the kit were coated with cytosine-rich DNA. DNMT can catalyze the binding of methyl group of S-adenosylmethionine onto cytosine of DNA. Methylated DNA can be recognized by anti-5-methylated cytosine antibody, and Enzyme-linked immunosorbent assay was performed to determine the amount of methylated DNA, which indirectly reflected methylation activity.

Analysis of Spl and NF- $\kappa B$ activities. After treatment with luteolin, nuclear proteins were extracted from the cells using protein extraction kit (Pierce). Bradford method was used to determine the concentrations of proteins. Activities of transcription factors $\mathrm{Sp} 1$ and $\mathrm{NF}-\kappa \mathrm{B}$ were determined according to the manufacturer's manual (EK1090 and EK1121; Affymetrix, Santa Clara, CA, USA). Primary antibodies of Sp1 and p65 were provided by the kits. Absorbance was determined at $450 \mathrm{~nm}$ to indirectly reflect the activities of Sp1 and NF- $\kappa$ B.

DNA extraction and hydrolysis. After treatment with luteolin, $1 \times 10^{7}$ BT474 or MCF-7 cells were used for the extraction of genomic DNA according to a previous report (17). Briefly, $200 \mathrm{ng}$ genomic DNA was denaturated at $100^{\circ} \mathrm{C}$ for $3 \mathrm{~min}$, before being cooled on ice. Then, $1 / 10$ volumes of ammonium acetate $(0.1 \mathrm{~mol} / 1, \mathrm{pH} 5.3)$ and $2 \mathrm{U}$ of nuclease P1 (US Biological, Swampscott, MA, USA) were added. After incubation at $45^{\circ} \mathrm{C}$ for $2 \mathrm{~h}, 1 / 10$ volumes of $\mathrm{NH}_{4} \mathrm{HCO}_{3}(1 \mathrm{~mol} / \mathrm{l})$ and $0.002 \mathrm{U}$ of snake venom phosphodiesterase I (Sigma-Aldrich) were added before incubation at $37^{\circ} \mathrm{C}$ for $1 \mathrm{~h}$. After addition of $0.5 \mathrm{U}$ alkaline phosphatase, the samples were incubated at $37^{\circ} \mathrm{C}$ for $1 \mathrm{~h}$. 

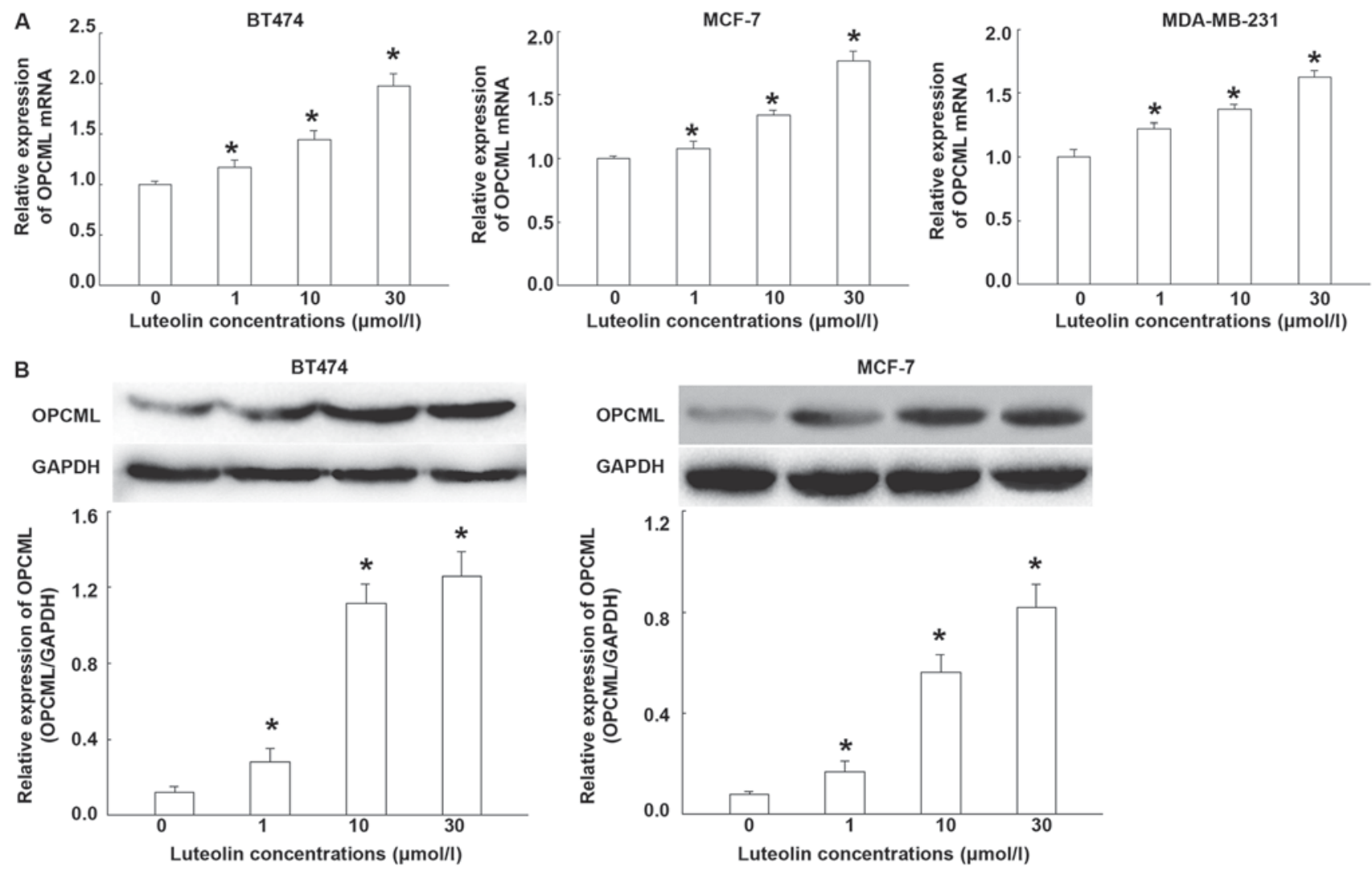

Figure 1.Effect of luteolin on the expression of OPCML in breast cancercells.(A) OPCML mRNA expression was determined by reverse transcription-quantitative polymerase chain reaction. (B) OPCML protein expression was determined by western blot analysis. The cells were treated with 0 (control), $5,10 \mathrm{and} 30 \mu \mathrm{mol} / 1$ luteolin. Each test was repeated for at least three times. ${ }^{*} \mathrm{P}<0.05$ vs. the control. OPCML, opioid binding protein/cell adhesion molecule.

High performance liquid chromatography-electrosprary ionization-mass spectrometry (HPLC-ESI-MS/MS). HPLCESI-MS/MS was performed using HPLC system (model 1100; Agilent, Santa Clara, CA, USA) and chromatographic column (Atlantis $\mathrm{dC}_{18}$ column), and pre-column (both from Waters, Milford, MA, USA). The mobile phase was $0.1 \%$ formic acid-methanol. Flow velocity was $0.2 \mathrm{ml} / \mathrm{min}$. Electrospray ionization mode was positive ion. Scanning range was $\mathrm{m} / \mathrm{z}$ $100-2,000$. Ion source temperature was $450^{\circ} \mathrm{C}$. Spray voltage was $415 \mathrm{kV}$, cluster voltage was $55 \mathrm{~V}$, and entrance voltage was $6 \mathrm{~V}$. Impact energy was $13 \mathrm{~V}$. Curtain gas pressure was $138 \mathrm{kPa}$, gas 1 pressure was $221 \mathrm{kPa}$, gas 1 pressure was $379 \mathrm{kPa}$, and collision gas pressure was $41 \mathrm{kPa}$ (17). Sciex Analyst software version 1.3.1 was used to analyze the data.

Lentiviral infection. About $10^{5}$ cells were inoculated in 6-well plates overnight. Before lentiviral infection, 293T cells were used to pack lentiviral vectors, and then BT474 or MCF-7 cells were inoculated into 24 -well plates at a density of $10^{5} /$ well. After $24 \mathrm{~h}$, Cells were transformed with lentivirus. On the first day of transformation, the medium was replaced with fresh medium containing $5 \mu \mathrm{g} / \mathrm{ml}$ polybrene, and then lentiviral particles were added until multiplicity of infection reached 10 . After incubation overnight, the medium was replaced with fresh complete medium, followed by addition of luteolin.

Flow cytometry. Cell cycle and DNA content of apoptotic cells were detected by flow cytometry after $24 \mathrm{~h}$ of drug exposure. Cells were collected and analyzed using flow cytometry. The number of cells was quantified by ModFit LT software (BD Biosciences, Franklin Lakes, NJ, USA).

Statistical analysis. The results were analyzed using SPSS 17.0 statistical software (SPSS, Inc., Chicago, IL, USA). The data were expressed as the mean \pm standard deviation. Comparison between groups was performed using analysis of variance with Dunnett's test as the post hoc test. Differences with $\mathrm{P}<0.05$ were considered statistically significant.

\section{Results}

Luteolin effectively upregulates the expression of OPCML in breast cancer cells. To test the effect of luteolin on the expression of OPCML mRNA and protein, RT-qPCR and Western blotting were performed. RT-qPCR showed that the levels of OPCML mRNA were increased with the increase of luteolin concentrations (Fig. 1A). Western blotting showed that luteolin treatment effectively increased protein expression of OPCML, being consistent with mRNA trend (Fig. 1B). In addition, the effect of luteolin on other breast cancer cells (MCF-7 and MDA-MB-231) were also tested and similar results were obtained. The results suggest that luteolin effectively upregulates the expression of OPCML in breast cancer cells.

Luteolin activates OPCML by reducing intracellular methylation levels. To determine the methylation level and activity, LC-MS/MS and EpiQuik TM DNMT1 detection 

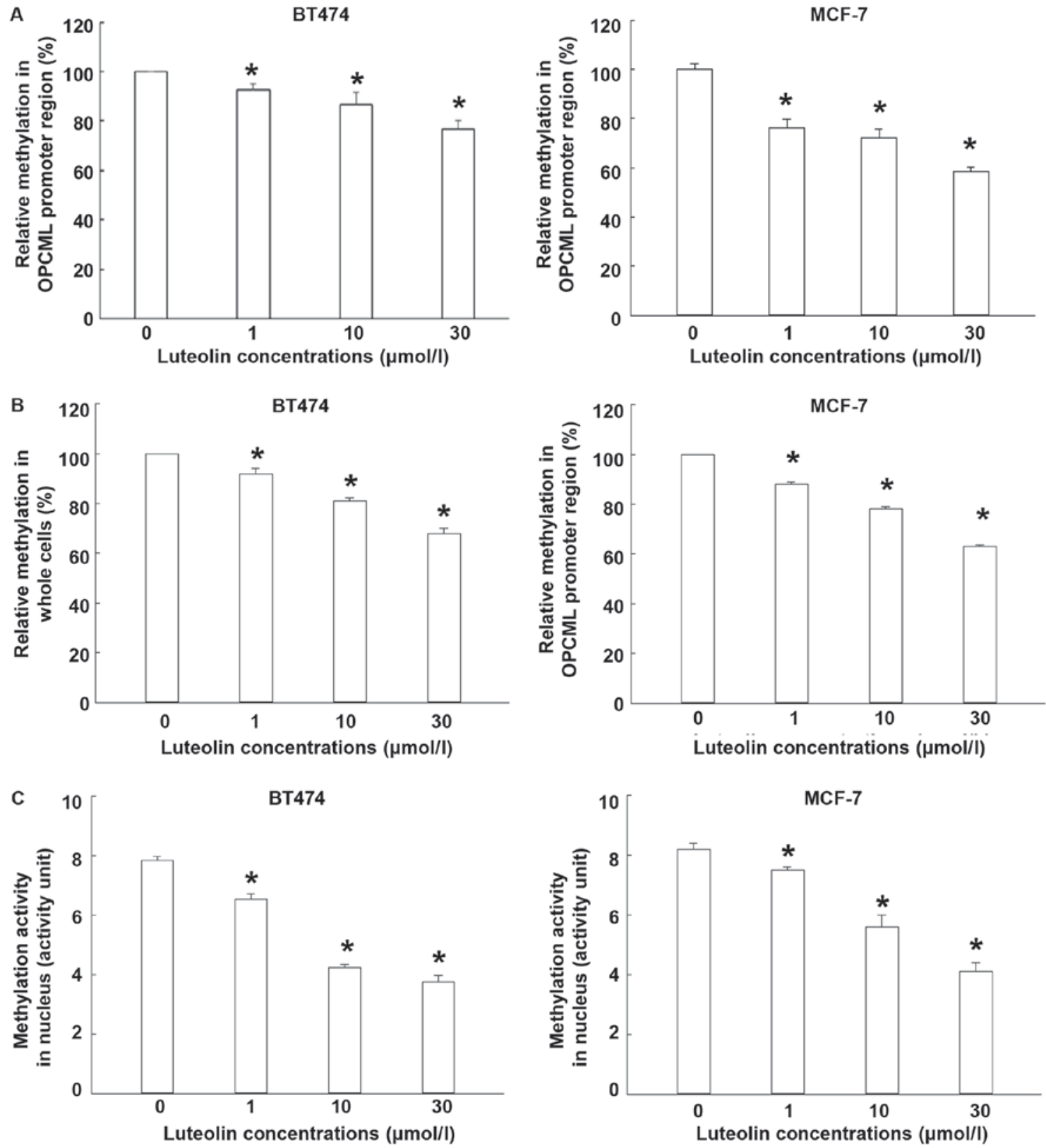

Figure 2. Effect of luteolin on methylation levels in the (A) OPCML promoter region, (B) whole cells and the (C) methylation activity in the nucleus. The cells were treated with 0 (control), 1, 10 and $30 \mu \mathrm{mol} / 1$ luteolin. LC-MS/MS was performed to determine the methylation levels and EpiQuik ${ }^{\mathrm{TM}}$ DNMT1 detection kit was used to determine methylation activity. Each test was repeated for at least three times. ${ }^{*} \mathrm{P}<0.05$ vs. the control. OPCML, opioid binding protein/cell adhesion molecule; DNMT1, DNA methyl transferase 1.

kit were used. The data showed that treatment with different concentrations of luteolin significantly decreased the methylation level of OPCML promoter region $(\mathrm{P}<0.05$; Fig. 2A). In the meantime, luteolin treatment reduced the global DNA methylation level compared with control $(\mathrm{P}<0.05$; Fig. 2B). Moreover, methylation activity of nucleoprotein in BT474 and MCF-7 cells were significantly reduced by luteolin treatment (Fig. 2C). The results indicate that luteolin activates OPCML by reducing intracellular methylation levels.

Luteolin downregulates intracellular methylation levels by decreasing Spl and NF- $\kappa B$ activities. To test whether luteolin affects the activities of Sp1 and NF-kB that regulate DNMT1 activity, ELISA and western blotting were used. ELISA showed that luteolin treatments significantly reduced the activities of Sp1 and NF- $\kappa \mathrm{B}(\mathrm{P}<0.05$; Fig. 3A and B). Western blotting showed that luteolin inhibited the expression of DNMT1 protein (Fig. 3C). The results suggest that luteolin downregulates intracellular methylation levels by decreasing Sp1 and NF- $\mathrm{KB}$ activities.

Luteolin affects the expression of DNMT1 and OPCML by downregulating Sp1 activity. To examine whether luteolin affects the expression of DNMT1 and OPCML via Sp1, we overexpressed Sp1 in BT474 and MCF-7 cells. The data showed that elevated expression of Sp1 attenuated the reduction of DNMT1 expression induced by luteolin. In the meantime, OPCML expression was inhibited by $\mathrm{Sp} 1$ overexpression (Fig. 4). The result indicates that luteolin affects the expression of DNMT1 and OPCML by downregulating Sp1 activity. 

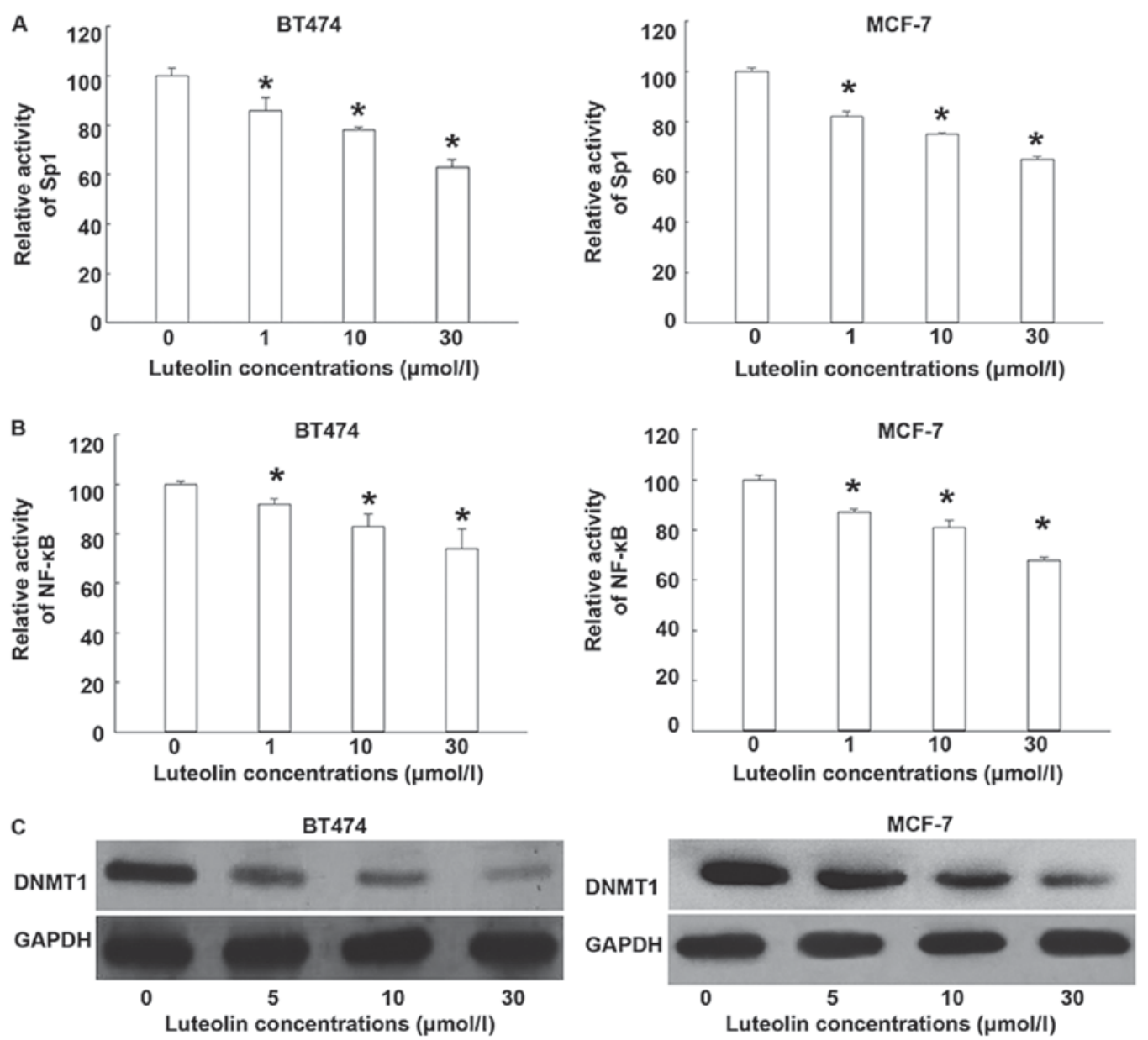

Figure 3. Effect of luteolin on the activity of the (A) Sp1 and (B) NF-kB transcription factors and the (C) expression of DNMT1. BT474 or MCF-7 cells were treated with 0 (control), 5, 10 and $30 \mu \mathrm{mol} / 1$ luteolin. ELISA was used to determine the activities of Sp1 and NF- $\kappa \mathrm{B}$, while western blot analysis was performed to measure the expression of DNMT1. Each test was repeated for at least three times. "P $<0.05$ vs. the control. NF, nuclear factor; DNMT1, DNA methyl transferase 1.
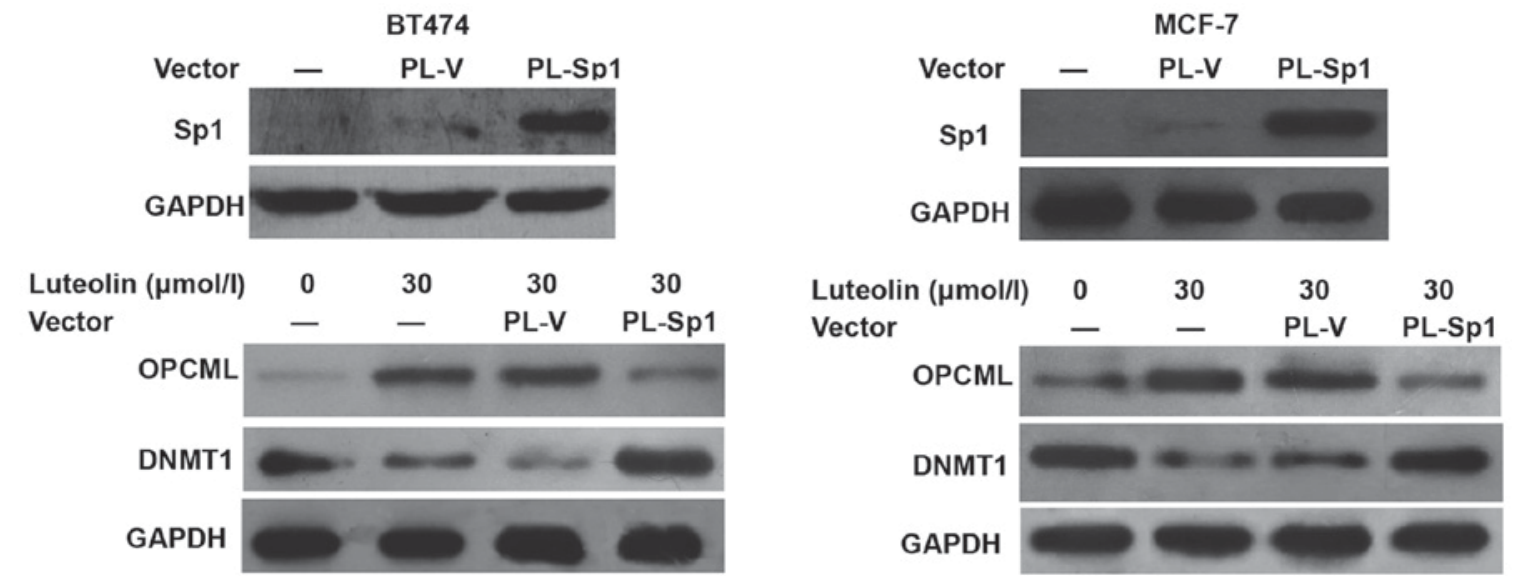

Figure 4. Effect of the expression of Sp1 on the expression of DNMT1 and OPCML. Cells were transfected with Sp1 lentiviral vector (PL-Sp1) or control vector (PL-V), and then incubated with $30 \mu \mathrm{mol} / 1$ luteolin for $72 \mathrm{~h}$. Western blot analysis was used to determine the transfection efficiency (upper) and expression of OPCML and DNMT1 proteins (lower). OPCML, opioid binding protein/cell adhesion molecule; DNMT1, DNA methyl transferase 1.

Luteolin inhibits the proliferation and induces the apoptosis of breast cancer cells. To evaluate the effect of luteolin on the proliferation of breast cancer cells, MTT assay and flow cytometry were carried out after treating the cells with different concentrations of luteolin. Cell cycle analysis showed that the percentages of BT474 and MCF-7 cells in G1 phase after treatment with luteolin were significantly increased, while the percentages of BT474 and MCF-7 cells in G2 phase were significantly decreased $(\mathrm{P}<0.05)$. In addition, the percentage of BT474 cells in $\mathrm{S}$ phase was significantly decreased $(\mathrm{P}<0.05)$ (Fig. 5A). MTT assay showed that luteolin inhibited the proliferation of BT474 and MCF-7 cells in a dose-dependent 
A

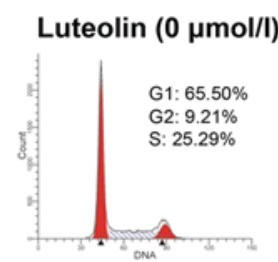

Luteolin $(10 \mu \mathrm{mol} / \mathrm{l})$

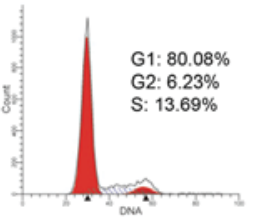

B

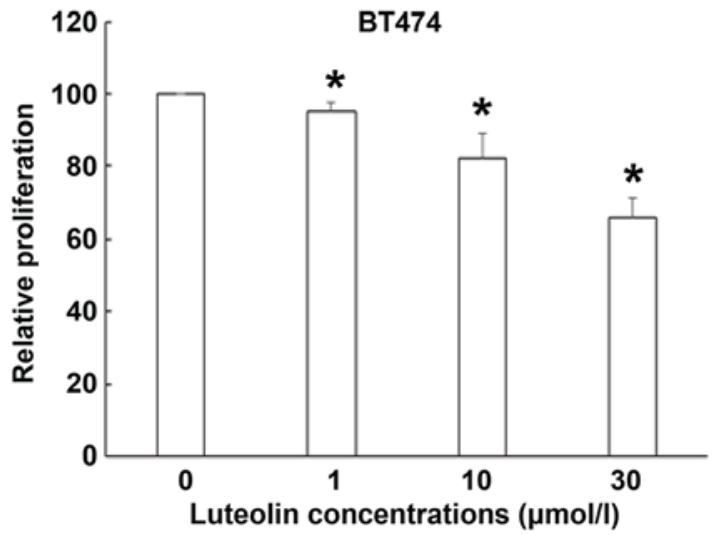

C

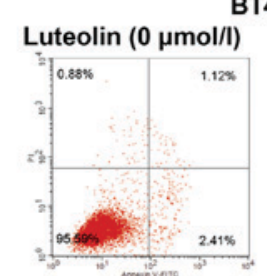

BT474
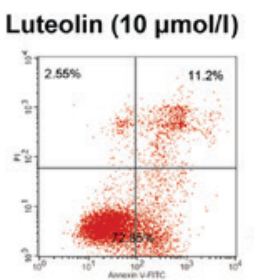

D

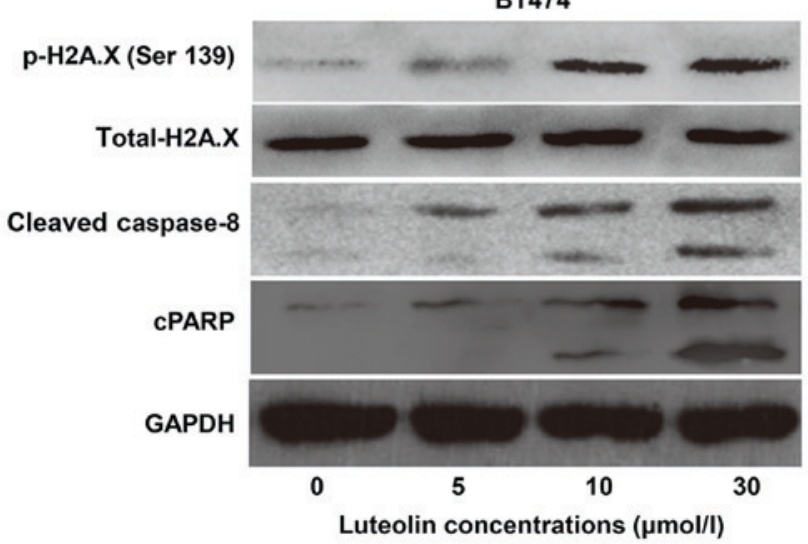

MCF-7

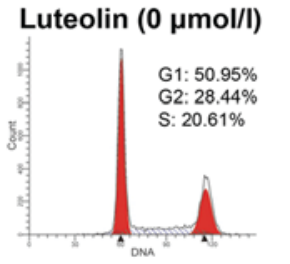

Luteolin (10 $\mu \mathrm{mol} / \mathrm{l})$
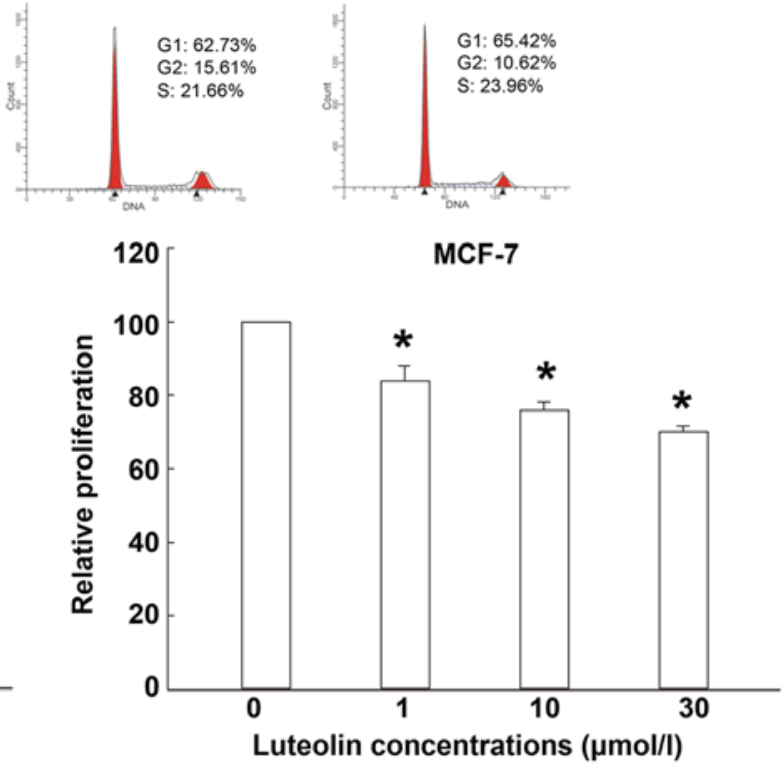

MCF-7

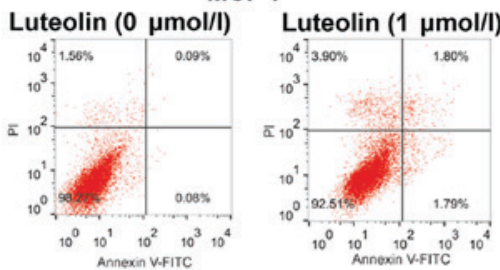

Luteolin $(\mathbf{1 0} \mu \mathrm{mol} / \mathrm{I}) \quad$ Luteolin $(30 \mu \mathrm{mol} / \mathrm{l})$

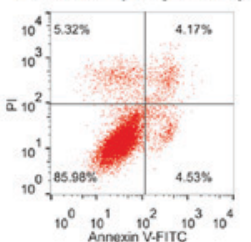

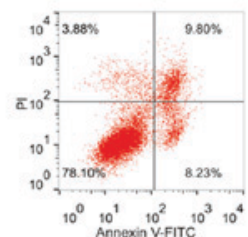

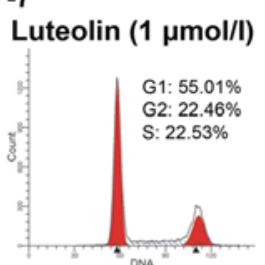

Luteolin $(30 \mu \mathrm{mol} / \mathrm{l})$

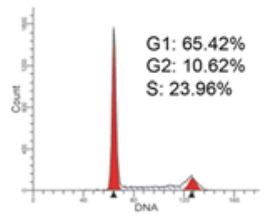

MCF-7

MCF-7

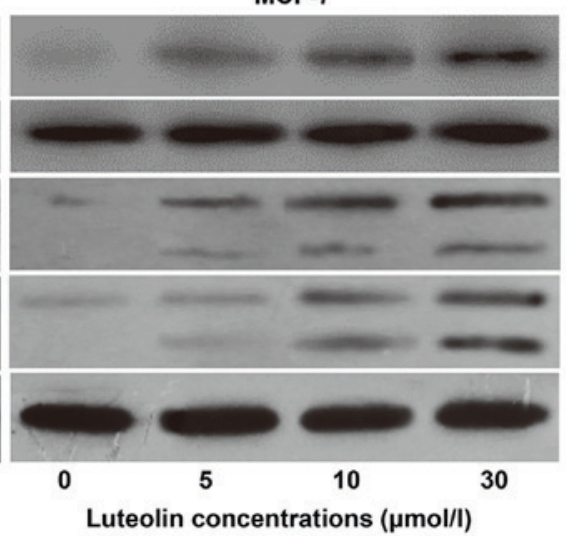

Figure 5. Effect of luteolin on the proliferation, cell cycle and apoptosis of BT474 and MCF-7 cells. (A) Cell cycle arrest of BT474 and MCF-7 cells. Cells were treated with 0 (control), 5,10 and $30 \mu \mathrm{mol} / 1$ luteolin for $72 \mathrm{~h}$. flow cytometry was used to evaluate the cell cycle arrest of the cells. (B) Relative proliferation of BT474 and MCF-7 cells. The cells were treated with 0 (control), 5,10 and $30 \mu \mathrm{mol} / 1$ luteolin for $72 \mathrm{~h}$. An MTT assay was used to evaluate the relative proliferation of the cells. Each test was repeated for at least three times. "P<0.05 vs. the control. (C) Apoptosis of BT474 and MCF-7 cells was detected by flow cytometry. (D) Phosphorylation of $\operatorname{H2AX}(\gamma-\mathrm{H} 2 \mathrm{AX})$, and activation of caspase-8 and PARP were determined by western blot analysis. cPARP, cleaved poly ADP-ribose polymerase. 
manner (Fig. 5B). Flow cytometry showed that luteolin induced apoptosis of BT474 and MCF-7 cells (Fig. 5C). To test whether luteolin induces DNA double-strand breaks and cell apoptosis, Western blotting was performed. The data showed that luteolin (5-30 $\mu \mathrm{mol} / \mathrm{l})$ significantly increased the expression of $\gamma$-H2AX (Ser139) and slicing of caspase- 8 and PARP (Fig. 5D). The results suggest that luteolin inhibits the proliferation and induces the apoptosis of breast cancer cells.

\section{Discussion}

Over the past half century, the incidence of breast cancer has steadily increased, but the mortality rate of breast cancer is relatively stable. This change is mainly due to the deepening of knowledge of breast cancer. Like other malignant tumors, breast cancer is the result of the activation of oncogenes and inactivation of tumor-suppressor genes. Hypermethylation of the promoter region of tumor-suppressor gene occurs at the early stage of malignant tumor formation, and is the most studied topic. DNA methylation is an important epigenetic mechanism of transcriptional regulation in eukaryotes. Changes in methylation patterns may promote the occurrence of tumors. OPCML gene is a tumor-suppressor gene discovered in recent years. It is a member of IgLON family that belongs to immunoglobulin superfamily. OPCML widely exists in nervous system cells, and plays important roles in regulating opioid receptors and signal transduction, promoting cell differentiation and changing cell membrane properties (18). It is reported that the expression of OPCML in a variety of malignant tumor tissues is deficient or low, and the promoter region of OPCML is usually methylated (19). In addition, the expression of OPCML is closely related to the recurrence, metastasis and prognosis of tumor patients (19).

Because of the reversibility of epigenetic changes, a new method of tumor therapy has been developed, and it has achieved some effect in the treatment of leukemia. Currently, DNA demethylation agents such as decitabine and azacytidine are already used in the clinical treatment of myelodysplastic syndrome (20). Luteolin is a natural flavonoid that is extracted from mignonette. The two adjacent polar $-\mathrm{OH}$ groups at $\mathrm{C} 3$ and $\mathrm{C} 4$ of luteolin benzene ring $\mathrm{B}$ are essential for the inhibition of enzyme activity. The conjugated double bond between $\mathrm{C} 2$ and $\mathrm{C} 3$ makes $\mathrm{B}$ rings and $\mathrm{C}$ rings in the same plane, being beneficial for approaching kinase substrate binding sites. These two kinds of structures are crucial for the inhibition of cell proliferation by luteolin $(21,22)$. Luteolin inhibits tumor cell growth factors by changing cell signaling pathways or resists cancer cell invasion by changing kinase activity (23). It can also inhibit tumor cell growth by blocking cell cycles (24). Our study on OPCML mRNA and protein expression shows that luteolin increases OPCML mRNA and protein expression in a dose-dependent manner, suggesting that luteolin induces the reexpression of OPCML by some unknown mechanism. Our data also show that the methylation status upstream of OPCML gene and in the cells is higher, and treatment with luteolin reduces the methylation level, suggesting that luteolin reverses the methylation status of OPCML gene in tumor cells, which is beneficial to its transcription and expression. The intracellular methylation activity is mainly related to DNMTs, so the decrease of intracellular methylation level after luteolin treatment may be caused by the inhibition of DNMTs expression or biological activity (25). Our data on DNMT1 expression and intracellular methylation show that luteolin downregulates the expression of DNMT1, and inhibits intracellular methylation activity. This may be a direct mechanism by which OPCML expression is upregulated.

$\mathrm{Sp} 1$ transcription factor belongs to the Sp protein family. $\mathrm{Sp} 1$ promotes the expression of a number of molecules that positively regulate cell cycle, such as cyclin D1, E2F1, c-fos, and TGF- $\alpha$, etc. In addition, Sp1 can also affect the methylation of $\mathrm{CpG}$ island of DNA. In a variety of tumor tissues, such as gastric cancer, pancreatic cancer, breast cancer and thyroid tumor tissues, Sp1 is often highly expressed, and its activity is positively correlated with the degree of tumor infiltration, and negatively correlated with the prognosis of patients $(26,27)$. In addition, $\mathrm{Sp} 1$ can also promote the expression of DNMT1 by forming a complex with NF- $\kappa \mathrm{B}$. Our data show that the activities of Sp1 and NF- $\kappa$ B in control group are high, and treatment with different concentrations of luteolin significantly reduces the activities of the two transcription factors. Yu et al (28) report that curcumin inhibits the expression of DNMT1 in human leukemia cells, possibly by inhibiting the activity of Sp1. Du et al (29) have shown similar results. In addition, overexpression of Sp1 in cells attenuates the inhibitory effect of luteolin on DNMT1 expression, and reverses the upregulating effect of luteolin on OPCML, suggesting that Sp1 affects the methylation and expression of OPCML by regulating intracellular levels of DNMT1. Sp1 expression is elevated in various tumor tissues, and correlated with poor prognosis. Therefore, targeted therapy against Spl activity can improve the prognosis of tumors to some extent, and luteolin has a beneficial effect on the tumors by affecting Sp1 activity.

For a variety of chemotherapeutic drugs, the inhibition of tumor cell proliferation and apoptosis is the ultimate goal. The main manifestations at the early stage of apoptosis are the phosphorylation of $\mathrm{H} 2 \mathrm{AX}(\gamma-\mathrm{H} 2 \mathrm{AX})$ and the activation of caspase family proteins (30). The $\gamma-\mathrm{H} 2 \mathrm{AX}$ is an important sign of early apoptosis, and the activation of Caspase family proteins can act on DNA repair enzyme, poly ADP-ribose polymerase (PARP), to deactivate it (31). Our study on the phosphorylation of $\mathrm{H} 2 \mathrm{AX}$ and the expression of PARP shows that luteolin treatment inhibits the proliferation of cells, promotes the phosphorylation of $\mathrm{H} 2 \mathrm{AX}$ and the activation of caspase, and induces the expression of PARP, suggesting that the inhibitory effect of luteolin on breast cancer cells may be achieved by inducing apoptosis. In conclusion, the present study suggests that luteolin inhibits the growth of breast cancer by decreasing the methylation and upregulating the expression of OPCML gene. Although additional experiments involving OPCML-silenced cells are required to further confirm that OPMLC mediates the antitumor effects of luteolin, there are a few pieces of evidence that support our hypothesis that inactivated OPCML by methylation is associated with multiple malignancies $(32,33)$. Its effect is similar with that of 5-aza-2-deoxycytidine. However, 5-aza-2-deoxycytidine may also inhibit human normal gene methylation when suppressing tumor gene methylation. This limits its clinical application. Luteolin is originated from natural plants. Although animal experiments show that luteolin has low toxicity $(34,35)$, its clinical application still 
remain to be studied. Therefore, the application values of luteolin in tumor chemotherapy will be further enhanced, if its structure is modified to reduce its effect on normal cells without compromising its antitumor activity.

\section{Acknowledgements}

Not applicable.

\section{Funding}

No funding was received.

\section{Availability of data and materials}

The datasets used and/or analyzed during the current study are available from the corresponding author on reasonable request.

\section{Authors' contributions}

XD, JianZ and JirenZ collaborated to design the study. FY, JW, $\mathrm{RC}$ and TW were responsible for performing the experiments. $\mathrm{XD}$ and JianZ analyzed the data. All authors collaborated to interpret the results and develop the manuscript. The final version of the manuscript was read and approved by all authors, and each author believes that the manuscript represents honest work.

\section{Ethics approval and consent to participate}

Not applicable.

\section{Consent for publication}

Not applicable.

\section{Competing interests}

The authors declare that they have no competing interests.

\section{References}

1. Sauvaget $\mathrm{C}$, Nishino $\mathrm{Y}$, Konno R, Tase $\mathrm{T}$, Morimoto $\mathrm{T}$ and Hisamichi S: Challenges in breast and cervical cancer control in Japan. Lancet Oncol 17: e305-e312, 2016.

2. Schoemaker MJ, Jones ME, Wright LB, Griffin J, McFadden E, Ashworth A and Swerdlow AJ: Psychological stress, adverse life events and breast cancer incidence: A cohort investigation in 106,000 women in the United Kingdom. Breast Cancer Res 18: 72, 2016.

3. Yan X, Han R, Zhou J, Yu H, Yang J and Wu M: Incidence, mortality and survival of female breast cancer during 2003-2011 in Jiangsu province, China. Chin J Cancer Res 28: 321-329, 2016.

4. Golubnitschaja O, Debald M, Yeghiazaryan K, Kuhn W, Pešta M, Costigliola V and Grech G: Breast cancer epidemic in the early twenty-first century: Evaluation of risk factors, cumulative questionnaires and recommendations for preventive measures. Tumour Biol 37: 12941-12957, 2016.

5. Lee JY and Kong G: Roles and epigenetic regulation of epithelial-mesenchymal transition and its transcription factors in cancer initiation and progression. Cell Mol Life Sci 73: 4643-4660, 2016.

6. Kasai H: What causes human cancer? Approaches from the chemistry of DNA damage. Genes Environ 38: 19, 2016.
7. Song Y, Wu F and Wu J: Targeting histone methylation for cancer therapy: ENzymes, inhibitors, biological activity and perspectives. J Hematol Oncol 9: 49, 2016.

8. Shui IM, Wong CJ, Zhao S, Kolb S, Ebot EM, Geybels MS, Rubicz R, Wright JL, Lin DW, Klotzle B, et al: Prostate tumor DNA methylation is associated with cigarette smoking and adverse prostate cancer outcomes. Cancer 122: 2168-2177, 2016.

9. Duarte-Pereira S, Paiva F, Costa VL, Ramalho-Carvalho J, Savva-Bordalo J, Rodrigues A, Ribeiro FR, Silva VM, Oliveira J, Henrique R and Jerónimo C: Prognostic value of opioid binding protein/cell adhesion molecule-like promoter methylation in bladder carcinoma. Eur J Cancer 47: 1106-1114, 2011.

10. Zhou F, Tao G, Chen X, Xie W, Liu M and Cao X: Methylation of OPCML promoter in ovarian cancer tissues predicts poor patient survival. Clin Chem Lab Med 52: 735-742, 2014.

11. Zhou F, Ma M, Tao G, Chen X, Xie W, Wang Y and Cao X: Detection of circulating methylated opioid binding protein/cell adhesion molecule-like gene as a biomarker for ovarian carcinoma. Clin Lab 60: 759-765, 2014.

12. Cui Y, Ying Y, van Hasselt A, Ng KM, Yu J, Zhang Q, Jin J, Liu D, Rhim JS, Rha SY, et al: OPCML is a broad tumor suppressor for multiple carcinomas and lymphomas with frequently epigenetic inactivation. PLoS One 3: e2990, 2008.

13. Liu CW, Lin HW, Yang DJ, Chen SY, Tseng JK, Chang TJ and Chang YY: Luteolin inhibits viral-induced inflammatory response in RAW264.7 cells via suppression of STAT1/3 dependent NF- $\kappa \mathrm{B}$ and activation of HO-1. Free Radic Biol Med 95: 180-189, 2016.

14. Wang L, Lee IM, Zhang SM, Blumberg JB, Buring JE and Sesso HD: Dietary intake of selected flavonols, flavones, and flavonoid-rich foods and risk of cancer in middle-aged and older women. Am J Clin Nutr 89: 905-912, 2009.

15. Gates MA, Tworoger SS, Hecht JL, De Vivo I, Rosner B and Hankinson SE: A prospective study of dietary flavonoid intake and incidence of epithelial ovarian cancer. Int J Cancer 121: 2225-2232, 2007.

16. Landis-Piwowar KR, Milacic V and Dou QP: Relationship between the methylation status of dietary flavonoids and their growth-inhibitory and apoptosis-inducing activities in human cancer cells. J Cell Biochem 105: 514-523, 2008.

17. Liu Z, Liu S, Xie Z, Blum W, Perrotti D, Paschka P, Klisovic R, Byrd J, Chan KK and Marcucci G: Characterization of in vitro and in vivo hypomethylating effects of decitabine in acute myeloid leukemia by a rapid, specific and sensitive LC-MS/MS method. Nucleic Acids Res 35: e31, 2007.

18. Sellar GC, Watt KP, Rabiasz GJ, Stronach EA, Li L, Miller EP, Massie CE, Miller J, Contreras-Moreira B, Scott D, et al: OPCML at $11 \mathrm{q} 25$ is epigenetically inactivated and has tumor-suppressor function in epithelial ovarian cancer. Nat Genet 34: 337-343, 2003.

19. Li C, Tang L, Zhao L, Li L, Xiao Q, Luo X, Peng W, Ren G, Tao Q and Xiang T: OPCML is frequently methylated in human colorectal cancer and its restored expression reverses EMT via downregulation of smad signaling. Am J Cancer Res 5: $1635-1648,2015$

20. Blum W and Marcucci G: Targeting epigenetic changes in acute myeloid leukemia. Clin Adv Hematol Oncol 3: 855-865, 882, 2005.

21. Guerrero L, Castillo J, Quiñones M, Garcia-Vallvé S, Arola L, Pujadas $\mathrm{G}$ and Muguerza B: Inhibition of angiotensin-converting enzyme activity by flavonoids: Structure-activity relationship studies. PLoS One 7: e49493, 2012.

22. Xu YC, Leung SW, Yeung DK, Hu LH, Chen GH, Che CM and Man RY: Structure-activity relationships of flavonoids for vascular relaxation in porcine coronary artery. Phytochemistry 68: 1179-1188, 2007.

23. Lin Y, Shi R, Wang X and Shen HM: Luteolin, a flavonoid with potential for cancer prevention and therapy. Curr Cancer Drug Targets 8: 634-646, 2008.

24. Seelinger G, Merfort I, Wölfle U and Schempp CM: Anti-carcinogenic effects of the flavonoid luteolin. Molecules 13: 2628-2651, 2008.

25. Jeltsch A: Molecular enzymology of mammalian DNA methyltransferases. Curr Top Microbiol Immunol 301: 203-225, 2006.

26. Sankpal UT, Goodison S, Abdelrahim M and Basha R: Targeting $\mathrm{Sp} 1$ transcription factors in prostate cancer therapy. Med Chem 7: 518-525, 2011.

27. Li L and Davie JR: The role of Sp1 and Sp3 in normal and cancer cell biology. Ann Anat 192: 275-283, 2010. 
28. Yu J, Peng Y, Wu LC, Xie Z, Deng Y, Hughes T, He S, Mo X, Chiu M, Wang QE, et al: Curcumin down-regulates DNA methyltransferase 1 and plays an anti-leukemic role in acute myeloid leukemia. PLoS One 8: e55934, 2013.

29. Du L, Xie Z, Wu LC, Chiu M, Lin J, Chan KK, Liu S and Liu Z: Reactivation of RASSF1A in breast cancer cells by curcumin. Nutr Cancer 64: 1228-1235, 2012.

30. Chiu SJ, Chao JI,Lee YJ and Hsu TS: Regulation of gamma-H2AX and securin contribute to apoptosis by oxaliplatin via a $\mathrm{p} 38$ mitogen-activated protein kinase-dependent pathway in human colorectal cancer cells. Toxicol Lett 179: 63-70, 2008.

31. Simbulan-Rosenthal CM, Rosenthal DS, Iyer S, Boulares H and Smulson ME: IInvolvement of PARP and poly (ADP-ribosyl) ation in the early stages of apoptosis and DNA replication. Mol Cell Biochem 193: 137-148, 1999.

32. Sellar GC, Watt KP, Rabiasz GJ, Stronach EA, Li L, Miller EP, Massie CE, Miller J, Contreras-Moreira B, Scott D, et al: OPCML at 11q25 is epigenetically inactivated and has tumor-suppressor function in epithelial ovarian cancer. Nat Genet 34: 337-343, 2003.
33. Cui Y, Ying Y, van Hasselt A, Ng KM, Yu J, Zhang Q, Jin J, Liu D, Rhim JS, Rha SY, et al: OPCML is a broad tumor suppressor for multiple carcinomas and lymphomas with frequently epigenetic inactivation. PLoS One 3: e2990, 2008.

34. Liu H, Zeng Z, Wang S, Li T, Mastriani E, Li QH, Bao HX, Zhou YJ, Wang X, Liu Y, et al: Main components of pomegranate, ellagic acid and luteolin, inhibit metastasis of ovarian cancer by down-regulating MMP2 and MMP9. Cancer Biol Ther 18: 990-999, 2017.

35. Katalinić M, Rusak G, Domaćinović Barović J, Sinko G, Jelić D, Antolović R and Kovarik Z: Structural aspects of flavonoids as inhibitors of human butyrylcholinesterase. Eur J Med Chem 45: 186-192, 2010. 